\title{
ENCEFALITIS POR VIRUS HERPES HUMANO TIPO 6 EN UNA ADOLESCENTE CON LUPUS ERITEMATOSO SISTÉMICO. PRIMER CASO REPORTADO EN EL ECUADOR
}

\author{
Del Valle Guerra Jhaneth ${ }^{1}$, Cruz Daniel Leonardo ${ }^{2 *}$, Merizalde Wendy Mercedes ${ }^{3}$, \\ Hidalgo Jaira Lorena ${ }^{3}$, Villacis Verónica Del Pilar ${ }^{4}$
}

DOI: 10.48018/rmv.v31.i2.16

Este artículo está bajo una licencia de Creative Commons de tipo Reconocimiento - No comercial - Sin obras derivadas 4.0 International.

1 Hospital de Especialidades Carlos Andrade Marín. Médico del Servicio de Infectología Pediátrica. Quito - Ecuador.

2 Hospital de Especialidades Carlos Andrade Marín. Médico del Servicio de Neurología Pediátrica. Quito - Ecuador.

3 Hospital de Especialidades Carlos Andrade Marín. Médico del Servicio de Pediatría. Quito - Ecuador. 4 Hospital de Especialidades Carlos Andrade Marín. Médico del Servicio de Reumatología. Quito - Ecuador.

ORCID ID:

Guerra Vilca Jhaneth

orcid.org//0000-0003-1088-7728

Cruz Montesinos Daniel

orcid.org/0000-0002-6417-9164

Merizalde Wendy Mercedes

orcid.org/0000-0001-7343-4561

Hidalgo Jaira Lorena

orcid.org/0000-0001-8127-6137

Verónica Del Pilar Villacis

orcid.org/0000-0003-3863-0705

*Corresponding author: Cruz Daniel Leonardo E-mail: dennis_cruz2002@yahoo.com

Article history

Received: 03 - Dic - 2020

Accepted: 11 - Ago - 2020

Publish: 01 - Nov - 2020

CARE 2017 Check List statement: The authors have real the CARE 2017 Check List and the manuscript was prepared and revised according to the CARE 2017 Checklist.

Conflict of interest: All authors declared that there are no conflicts of interest.

Financial disclosure: The authors have no financial relationships relevant to this article to disclose

Authors' contribution: All the authors contributed in the search, selection of articles and writing. All the authors reviewed and approved the final manuscript.

Forma de citar este artículo: Del Valle Guerra J, Cruz DL, Merizalde WM, Hidalgo JL, Villacis V. ENCEFALITIS POR VIRUS HERPES HUMANO TIPO 6 EN UNA ADOLESCENTE CON LUPUS ERITEMATOSO SISTÉMICO. PRIMER CASO REPORTADO EN EL ECUADOR. Rev Med Vozandes. 2020; 31 (2): 114 - 116

\section{Resumen}

Se describe el caso de una paciente de catorce años con Lupus Eritematoso Sistémico (LES) con índice de actividad severo y en tratamiento inmunosupresor. Acudió al servicio de Urgencias Pediátricas del Hospital de Especialidades Carlos Andrade Marín, por presentar fiebre, cefalea, náuseas, alucinaciones auditivas y paresia en extremidades inferiores. Se realizó estudio de líquido cefalorraquídeo por Reacción en Cadena de la Polimerasa, en el cual se detectó la presencia de Virus Herpes Humano tipo 6 o también llamado Roseolovirus. Se inició ganciclovir con respuesta clínica favorable en 72 horas. Conclusiones: Se debe considerar la presencia de encefalitis por HHV-6 en pacientes inmunocomprometidos con encefalopatía y el uso de ganciclovir como terapia dirigida.

Palabras clave: Lupus eritematoso sistémico, Virus Herpes Humano 6, encefalitis

\section{Abstract \\ FIRST CASE REPORTED IN ECUADOR: HUMAN HERPES VIRUS TYPE 6 ENCEPHALITIS IN ADOLESCENTS WITH SYSTEMIC LUPUS ERYTHEMATOSUS. FIRST CASE REPORTED IN ECUADOR.}

The case of a fourteen-year-old patient with Systemic Lupus Erythematosus with severe activity index and immunosuppressive treatment is described. He went to the Pediatric Emergency Department of the Carlos Andrade Marín Specialty Hospital, for presenting fever, headache, nausea, auditory hallucinations and paresis in the lower extremities. Cerebrospinal fluid was studied by Polymerase Chain Reaction, in which the presence of Human Herpes Virus type 6 or also called Roseolovirus was detected. Ganciclovir was started with a favorable clinical response in 72 hours. Conclusions: The presence of HHV-6 encephalitis should be considered in immunocompromised patients with encephalopathy and use of ganciclovir as directed therapy.

Keywords: Systemic Lupus Erythematosus, Human Herpes Virus 6, Encephalitis 


\section{Introducción}

El Virus Herpes Humano (HHV-6), es un virus ubicuo DNA de doble cadena que pertenece al género Roseolovirus ${ }^{1,2}$. Según sus características genéticas y fenotípicas ha sido clasificado en dos especies distintas HHV-6A Y HHV-6B, son genéticamente homólogas al citomegalovirus8. El HHV-6, ha sido ampliamente observado en la población general, especialmente en el continente asiático. Infecta a casi todos los niños, teniendo una seroprevalencia mayor al $95 \%^{3}$.

La mayoría de las infecciones por HHV-6 son asintomáticas. La primo infección usualmente se asocia a exantema súbito, también conocido como roséola infantil o sexta enfermedad. Suele presentarse en infantes de entre seis meses a tres años; e inicia con fiebre auto limitada durante tres a cinco días asociados a crisis epilépticas, posteriormente cuando la temperatura cede aparece exantema rubeoliforme en cuello y tórax que dura entre uno a dos días ${ }^{1,2}$. En raros casos pueden existir complicaciones tales como: infecciones respiratorias, síndromes digestivos, meningoencefalitis, falla hepática y síndrome de activación de macrófagos ${ }^{1,3}$.

Al igual que otros virus herpes, luego de la primo infección los virus permanecen latentes en el organismo, pudiendo reactivarse de manera asintomática 0 asociado a síndromes clínicos de severidad variable y que usualmente ocurre en estados de inmunosupresión sea esta de origen farmacológico o adquiridas ${ }^{1,3}$. Las presentaciones clínicas más comunes son: encefalitis, fiebre, exantema, citopenia, neumonitis, hepatitis.

Existen reportes de encefalitis por reactivación del HHV6 en pacientes inmunocomprometidos, especialmente niños o adultos de veinte o treinta años de edad después de trasplante de células madres hematopoyéticas; de igual manera las neoplasias hematológicas, los trasplantes alogénicos, y el trasplante de células del cordón umbilical se han identificado como los principales factores de riesgo ${ }^{2,3}$.

La infección por HHV-6 ha sido cada vez más reconocida como una complicación grave en pacientes inmunocomprometidos ${ }^{3}$. A continuación se presenta un caso de encefalitis por reactivación del HHV-6 en una paciente con Lupus Eritematoso Sistémico (LES) con tratamiento inmunosupresor.

\section{Caso clínico}

Se trata de paciente adolescente, femenino, de catorce años de edad con antecedente patológico personal de Lupus Eritematoso Sistémico con manifestación de neurolupus y nefritis lúpica grado I, catalogado por reumatología con índice de actividad severo y en tratamiento inmunosupresor (corticoide). La paciente quince días previos al ingreso a hospitalización presentó artralgias y astenia; en un primer momento fue manejada de manera ambulatoria con aines (diclofenaco por diez días), posteriormente se asoció al cuadro alza térmica cuantificada de 38,5 a 39 grados centígrados, cefalea, náusea, alteración del estado de alerta, alucinaciones auditivas simples y disminución de fuerza muscular. En su ingreso a hospitalización, se evidenció: lesiones ulceradas en mucosa oral y paresia a predominio de miembros inferiores. Bajo sospecha clínica de encefalopatía aguda de causa infecciosa se realizó punción lumbar, reportándose en el líquido cefalorraquídeo hiperproteinorraquia e hipoglucorraquia, además se realizó estudio por reacción en cadena de la polimerasa (PCR) detectando la presencia de HHV6. Se inició terapéutica antiviral con ganciclovir con el cual se obtuvo adecuada respuesta clínica a las setenta y dos horas con desaparición de alucinaciones auditivas, fiebre y cefalea.

\section{Discusión}

La etiología de Lupus Eritematoso Sistémico no está clara, es una enfermedad muy heterogénea, tanto en sus manifestaciones clínicas como en su curso clínico, según lo describe la Sociedad Americana de Reumatología. ${ }^{16}$

El debut de un cuadro de encefalitis por HHV-6 en una paciente con LES inmunosuprimida, planteó la necesidad de conocer una posible relación - asociación clínica entre las dos entidades. En el lupus eritematoso sistémico se reporta mayor prevalencia de infección activa por HHV-6 (36.4\%)8, estos hallazgos sugieren que los pacientes con enfermedades autoinmunes, especialmente LES presentan una replicación activa del HHV6, teniendo una asociación significativa entre la actividad de la enfermedad y la viremia por HHV6. En la literatura médica, se refiere que tanto el HHV-6A como el HHV-6B están implicados como agentes etiológicos o cofactores para una amplia gama de afecciones patológicas en el sistema nervioso central (SNC); diferentes formas clínicas de encefalitis, encefalopatía, epilepsia, convulsiones febriles, meningitis, esclerosis múltiple ${ }^{8,9}$.

En una paciente con LES inmunosuprimida como el presente caso, nos cuestionamos si fue la inmunosupresión la que reactivo al HHV6. En el estudio de Brocolo y cols se encontró una asociación significativa entre la reactivación de HHV-6 y el estado activo de la enfermedad solo para el lupus eritematoso ( $P=0.021)$, lo cual sugiere que el HHV-6 puede actuar como un factor patogénico que predispone a los pacientes al desarrollo de enfermedades autoinmunes; o por el contrario, que estos trastornos pueden predisponer a los pacientes a la reactivación del HHV-67,8,9, 10. Por lo cual se plantea la hipótesis: el HHV-6, puede predisponer al desarrollo de lupus eritematoso sistémico y, de manera inversa, el LES puede actuar como un factor predisponente para la reactivación del HHV-6.

Otra manera de relacionar la encefalitis por el HHV-6 y el LES en nuestro caso, es quizás el uso de hidrocortisona y prednisona, los cuales pueden actuar como potente potenciador de la replicación de HHV-6 pudiendo favorecer el aumento de la carga viral ${ }^{11,12}$

El diagnóstico de la infección por HHV-6 se basa en la detección directa del virus o sus componentes en sangre, plasma, saliva, LCR; sin embargo la identificación de infección del HHV-6 a nivel del 
sistema nervioso central puede ser por PCR y por cultivo, esta última está siendo reemplazado por la detección de ácidos nucleicos específicos por PCR y el hallazgo de ADN de HHV-6 2, 3,11, 12, 13. En el Ecuador previamente no se habían reportado casos de encefalitis por HVV-6 en LES, quizás una de las explicaciones sea los pocos años de la técnica de PCR en el país.

La primo infección por roseolovirus usualmente no requiere tratamiento específico, sin embargo, cuando la reactivación de HHV-6 es reconocida en un paciente con inmunosupresión, se debe iniciar medicación anti roseolovirus, en nuestro caso se cumplió con esta recomendación. El ganciclovir es el tratamiento antiviral ampliamente recomendado para una encefalitis por HVV-6 ${ }^{15}$. A pesar de no estar incluido en el Cuadro Básico Nacional de medicamentos, por evidencia médica y experiencia clínica de infectología pediátrica se decidió su uso, obteniendo excelentes resultados con remisión de clínica neurológica

\section{Conclusiones}

La evidencia sugiere la participación del HHV-6 en pacientes con LES; poco se escribe sobre el tema en América Latina, la mayoría de los estudios son en el continente asiático y europeo. El HHV-6 debe ser considerado entre las etiologías de encefalitis en pacientes con LES. El ganciclovir es el tratamiento antiviral de elección, con una respuesta clínica rápida y favorable.

\section{Abreviaturas}

LES: Lupus Eritematoso Sistémico

LCR: líquido cefalorraquídeo

HHV-6A: virus herpes humano $6 \mathrm{~A}$

HHV-6B: virus herpes humano 6B

PCR: Reacción en cadena de polimerasa

\section{Agradecimientos}

Al área de Pediatría y Reumatología del Hospital de Especialidades Carlos Andrade Marín, por el manejo multidisciplinario del caso.

\section{Referencias}

1. Santoro JD, Hemond CC. Human herpesvirus 6 associated post-transplant acute limbic en6 associated post-transplant acute limbic encephalitis: Clinical observations of biomarkers
for risk of seizure in a pediatric population. Transpl Infect Dis. 2019 Feb;21 (1): e13003. doi: 10.1111 /tid.13003. Epub 2018 Oct 15. PubMed PMID: 30256500.

2. Hoffmann A, Kirn E, Kuerten A, Sander C Krueger GR, Ablashi DV. Active human herKrueger GR, Ablashi DV. Active human her-
pesvirus-6 (HHV-6) infection associated with pesvirus-6 (HHV-6) infection associated with
Kikuchi-Fujimoto disease and systemic lupus erythematosus (SLE). In Vivo. 1991 MayJun;5(3):265-9. PubMed PMID: 1654149.

3. Green DA, Pereira M, Miko B, Radmard S Whittier S, Thakur K. Clinical Significance of Human Herpesvirus 6 Positivity on the FilmAray Meningitis/Encephalitis Panel. Clin Infect Dis. 2018 Sep 14;67(7):1125-1128. doi: 10.1093/ cid/ciy288. PubMed PMID: 29635334.

4. Duménil J, Galambrun A, Basset et al. Human herpes virus-6 encephalitis in a paediatric bone marrow recipient: Successful treatment with pharmacokinetic monitoring and high doses of ganciclovir. Bone Marrow Transplant. 2006; (38): :769-770.

5. Linde A, Fridell E, Dahl $\mathrm{H}$ et al. Effect of primary Epstein-Barr virus infection on human herpesvirus 6, cytomegalovirus, and measles virus immunoglobulin G titers. J Clin Microbiol. 1990: 28:211-215.

Agut, H., Collandre, H., Aubin, J.T. et al, In vitro sensitivity of human herpesvirus-6 to antivira drugs. Res Virol. 1989; (140) :219-228.

7. Long, M.C., Bidanset, D.J., Williams, S.L., Kushner, N.L., Kern, E.R. Determination of antiviral efficacy against lymphotropic herpesviruses utilizing flow cytometry. Antiviral Res. ruses utilizing flow
2003:58:149-157.

8. Steiner I, Budka H., Chaudhuri A. et al. Viral encephalitis: A review of diagnostic methods and guidelines for management. Eur J Neurol. 2005;(12):331-343.

9. Ablashi D, Agut H, Alvarez L, et al . Classifica tion of HHV-6A and HHV-6B as distinct viruses. Arch Virol . 2014; (159):863-870.

10. Broccolo F, Drago F, Cassina G, Fava A, et al. Selective reactivation of human herpesvirus 6 in patients with autoimmune connective tissue diseases. J Med Virol.2014:85(11):192.

11. Yamamoto W, Ogusa E, Matsumoto K, Maruta A, Ishigatsubo Y, Kanamori H. Human herpesvirus-6 encephalopathy after hematopoietic stem cell transplantation and class I human leukocyte antigen. Clin Transplant 2014 ;(28):540-545

12. Yamamoto S, Takahashi S, Tanaka R, Okaya ma A, Araki A, Katano H, Tanaka-Taya K, AzU ma $\mathrm{H}$ (2015) Human herpesvirus-6 infectionassociated acute encephalopathy without skin rash. Brain Dev.2014; 3( 8): 829-832.

13. Krueger GR, Sander C, Hoffmann A, Barth $A$, Koch B, Braun M. Isolation of human herpesvirus-6 (HHV-6) from patients with collagen vascular diseases. In Vivo. 1991 MayJun;5(3):217-25. PubMed PMID: 1654147.

14. Adams MJ, Carstens EB. Ratification vote on taxonomic proposals to the International Committee on Taxonomy of Viruses. Arch Virol. 2012;157:1411-1422. doi: 10.1007/s00705rol. 2012;157:1

15. Agut $H$. Deciphering the clinical impact of acute human herpesvirus 6 (HHV-6) infections. J Clin Virol. 2011;52:164-171. doi: 10.1016/j.jcv.2011.06.008.

16. S. Bernatsky, J. Boivin, L. Joseph, S. Manzi, E. Ginzler, D. et al. Mortality in systemic lupus erythematosus. Arthritis Rheum; 54 :(2006) 2550-2557 